\title{
Information and ideas: concept design in three industrial contexts
}

\author{
Andrew Wodehouse and William Ion \\ Department of Design, Manufacture \& Engineering Management (DMEM), \\ University of Strathclyde, Glasgow, UK
}

\begin{abstract}
This paper reviews the application of a new concept design method in a number of industrial settings. The ICR Grid is intended to better integrate information into the concept design process. In addition to sketching and sharing concepts in a manner similar to the 6-3-5 Method, participants undertake information search tasks, use specific information items for concept development, and reflect on the merit of concepts as the session progresses. Three different companies were invited to utilise the method to address current design issues. Grid output, observation and semi-structured interviews were used to assess the performance of the method, with marked differences in use across organisations highlighting future potential applications and development.
\end{abstract}

\section{Keywords}

collaborative design; digital information; conceptual design; design method

\section{Introduction}

Concept design is the process undertaken when trying to develop solutions for a given problem, and covers the generation of ideas through to the selection of an embodied concept. Associated activities are often undertaken by groups in a collaborative setting and despite the fact this is typically a fuzzy process based around sketch work and discussion, a number of formal tools and techniques have been developed to support the process (Cross, 1994, French, 1985, Pahl and Beitz, 1995, Pugh, 1991, Ulrich and Eppinger, 1995). Although it has been observed that exposure to previous solutions can in some cases lead to fixation on particular approaches (Smith et al., 2008), access to appropriate information, principles, exemplars and context have been shown to be important in creating well-substantiated concepts and acting as stimuli for discussion (Benami and Jin, 2002, Chuang and Chen, 2008). Approaches such as storytelling (Demian and Fruchter, 2009), metaphors (Casakin, 2007) and inquiry (Eris, 2004) have been explored in recent studies as a means to enhance use of information in concept 
design. Despite investigation these structured approaches, however, brainstorming and informal collaboration in various forms (Sutton and Hargadon, 1996) remains a popular way for designers to exchange ideas in a non-critical environment and rapidly produce conceptual output. This presents a challenge in effectively integrating information into the concept design activity without prescribing a highly systematic approach.

In terms of basic cognitive processes, concept design is often divided into three phases. Osborn (1953) describes the creative problem-solving process of comprising: fact finding (problem definition and preparation), idea finding (thinking up ideas and leads) and solution finding (evaluation and adoption). Similarly, Cross (1994) argues prescriptive processes tend to follow a basic structure of analysis-synthesis-evaluation where analysis addresses all the design requirements for a problem, synthesis addresses solutions for each performance specification and evaluation addresses the accuracy with which these meet the requirements. Sim and Duffy (2003) identify a set of generic design activities numbering 27, but still categorise these three main aspects. It has been suggested (Cross, 1994, Dorst and Cross, 2001) that shifting between these modes in a flexible way can be beneficial, given the designer's tendency to make 'rapid explorations of problem and solution in tandem, in the coevolution of problem and solution' (Cross, 2004) rather than follow linear stages. Goldschmidt (1991) has made similar observations regarding the sketching, emphasizing the importance of 'shifts in perception' that occur during this activity in terms of creativity and the development of novel design solutions. Restrepo and Christiaans (2004) further explore problem/solution focusing strategies in design, arguing that designers are often solution-led rather than problem-led, and concluding that information and its accessibility are critical in supporting this activity:

Even when information exists and is relevant, it would not be used if its source were perceived as inaccessible. These are good reasons to make information tools more accessible to designers and, why not, fun to use! (p.12)

\section{Development of method}

As a result, the ICR Grid (the name derived from the Inform, Create, Develop cognitive processes and resulting grid output) has been developed. This can be viewed as a further development of the line of progressive techniques (Shah et al., 2000) including the 6-3-5 
Method (Rohrbach, 1969), the Gallery Method (Hellfritz, 1978) and C-Sketch (Kulkarni et al., 2001). These all rely on the basic principle of sharing concept sketches to stimulate ideas. The ICR Grid differs by incorporating information retrieval in order to help build information context and provide design stimuli. Another major addition is the competitive element introduced through the use of evaluation - after a concept has been created, it is passed on to the next participant who reflects on whether the idea is worth developing further. If a positive decision is made, a new information resource is found to apply to the concept and added to the library. If a negative decision is made a new concept is created. This cycle continues for a number of rounds, creating a grid of information and ideas linked by the actions taken during the session.

\subsection{Performance of the method}

The format and use of the method is outlined in Figure 1. At the beginning of each round, participants review the square above. If it contains an information item, this should be used as inspiration or stimulus in the development of a concept. As threads evolve, concepts are expected to become more detailed with the consistent introduction of information and new ideas to augment the established direction. If the square above contains a concept, this must be reviewed and a decision made on whether to develop it further. If the decision is positive, a new, relevant information item with suggested application must be sourced and inserted into the grid. If the decision is negative, a new, alternative concept is created.

The combination of linked information sources and concepts in the grid output is unique. The number of columns correlates to the number of participants involved, with each column forming a thread. This means that if positive decisions are consistently made then a concept can evolve with the continual addition of relevant information. Additionally, each thread has a different focus derived from the design problem to encourage concept diversity. Participants complete squares of the grid according to the flowchart, and each time a round is completed move down to the next row and across to the adjacent square. This ensures participants are exposed to all the information and ideas produced by others in the team.

The effectiveness of the method was been previously examined using a cohort of MSc students in a comparative study with the 6-3-5 Method (Wodehouse, 2010). Using Shah's metrics for concept design (Shah and Vargas-Hernandez, 2003), it was found that the ICR Grid performed better in terms of producing concepts of superior quality, variety and detail 
(Figure 2). In addition, the method was well received by participants and was found to be effective in bringing information to bear on concept design.

\section{Industrial application}

In order to examine the flexibility of the method when used in a variety of real-life contexts, a number of industrial tests were conducted. The setup for the sessions (Figure 3) used Microsoft OneNote (http://office.microsoft.com/en-us/onenote) to share digital information. This provided a live, shared document that all participants could simultaneously access and update. Tablet interfaces were used for sketching, allowing the sessions to take place entirely in the OneNote environment. The researcher was therefore present in the room and active in the digital environment to ensure that the format of the board was consistent and to deal with any technical issues. To help clarify the status of the shared board, the researcher's laptop was connected to a projector, providing a reference point and allowing participants to monitor any discrepancy between their board and the latest shared update. It also provided an easily legible version of the board and a shared visual focus for the sessions.

The sessions themselves took place over half a day each, including an introduction to the method, tutorial on the technology used, and debrief, with the actual design work roughly an hour in duration. Since previous studies had established the effectiveness of the method in improving conceptual output, these tests were used to develop an understanding of the process of using the method and its practicality in the industrial setting. The output grids were therefore reviewed for the number of rounds completed, concepts created and information sources found rather than the concepts themselves. The results are summarised in Table 1 and explored in more detail below. In addition, qualitative data from the semi-structured interviews and observation were used to evaluate the reaction of participants.

\subsection{Case 1: LAT56 $^{\circ}$}

LAT56 ${ }^{\circ}$ (http://www.lat56.com) design and manufacture 'technical business travel luggage'. Founded in 2007 and consisting of two designers, they have designed and patented a range of luggage products including the Rat-Pak ${ }^{\mathrm{TM}}$, a compact suit-carrier to allow easy transportation in demanding situations such as cycling. 


\subsubsection{Approach to concept generation}

Given the design background of the partners in the company, it is unsurprising that LAT56 ${ }^{\circ}$ have a high awareness of the product development process and the place of structured techniques in supporting it. They use a systematic brainstorming approach to tackle design challenges, with sessions often lasting over several days and progressing from words to ideas to concepts.

The design problem LAT $56^{\circ}$ chose to address was a current issue they had with their RatPak product. It was necessary to develop an integrated device which would allow the unfolded suit carrier to be hung over a rail or door. This would have to fit within the current space envelop of the product, be flexible enough to fit over several types of rail or door, and be as cheap to manufacture as possible.

\subsubsection{Session output}

The output from the session is shown in Figure 4. Eight rounds were completed in the session, which lasted just over an hour. The first two took almost ten minutes each, but it sped up significantly thereafter. Despite teams as small as two had not been considered when developing the method, the mechanics remained viable in this format. It was found, however, that the two resulting threads developed broadly similar concepts - a concept using a loop of Velcro or similar fastening material - with the dyad an attributable factor. With only two initial information sources, and lack of other participants bringing diverse information sources and ideas as the session progressed, there was limited scope for a range of information and ideas to be introduced and developed.

The strengths of the ICR Grid with regards to integrating information and concept development were apparent in Rounds 5-8, as a number of manufacturers and suppliers of components to allow different configurations of the basic design principle were established and explored. This supported a level of output appropriate for a product and problem approaching the manufacturing stage and illustrated how the grid could be used as a progressive concept development tool.

\subsection{Case 2: Scottoiler}

Scottoiler (http://www.scottoiler.com) manufacture chain lubrication systems for motorcycles. Their vacuum-operated chain lubrication system enables the chain to be cleaned and 
lubricated continuously while the engine is on by using a reservoir system mounted on the bike which slowly releases the oil. Scottoiler kits give a range of approximately 400-800 miles between refills of the RMV (Reservoir Metering Valve), depending on the flow setting, with a range of fittings suitable for most bikes, with the supplied bottle of Scottoil sufficient for 2500 miles of lubrication. The company was founded in 1986 and now employs 23 people, with the R\&D team consisting of three Design Engineers and a R\&D Project Manager.

Scottoiler have a range of established products, meaning much of their work is on incremental improvement and problem solving. Additionally, the on-site manufacturing issues can result in a lot of time and effort being absorbed by production and customer-related issues. In terms of their design and development process, concerted innovation generally takes place in the form of informal brainstorming sessions as part of their periodic R\&D team meetings. These utilise whiteboards and discussion to produce ideas, with consensus generally being used to dictate direction. Occasionally, $R \& D$ team members will take different concepts resulting from these sessions to embody individually and bring them back to the team for evaluation.

\subsubsection{Design problem}

The design problem addressed in the session was a generic one: how to improve delivery of oil to motorcycle chains. It was viewed by the R\&D team as an opportunity to encourage internal collaboration and develop new lines of thinking. Fitting, delivery and reliability were identified as the main criteria for any new design. Given the problems with a limited initial information base inhibiting the previous session with LAT56 $6^{\circ}$, the first row of the ICR Grid was filled by the researcher with a diverse (and random) range of sources prior to Scottoiler's session. This was intended to ensure that the four threads would lead to a heterogeneous set of concepts.

\subsubsection{Session output}

The output from the session is shown in Figure 5. Although the completed first row helped ensure the session started and continued at a reasonable pace (in all, eight rows of the grid were completed in the hour), it was became apparent that the team were uncomfortable with some of the directions the initial information items forced them into. Nevertheless, as the 
participants developed an understanding of the grid method, a diverse range of information items and concepts began to emerge in Rounds 2-5. The team size of four was found to be more effective than the two in the previous session, with the threads providing a variety of topics for individuals to address. This seemed to help with levels of engagement and information exchange.

The team had generally good levels of IT and sketching ability (though one participant did struggle more than the others), meaning that they were able to cope with the OneNote interface and tablet equipment necessary for the integrated environment. In terms of information items, catalogue parts and images of components from other manufacturers featured highly, accompanied by suggestions or ideas on how these could be applied in the chain lubrication context. The decisions made during the session were mostly positive, though there were a couple of no's. Again, the decision seemed to matter less than moving the idea or though forward in some way. Towards the end of the session, the participants seemed to find the general direction of Thread D (which addressed mudguard configuration) the most exciting in terms of its development, though there were elements of cross-fertilisation across the columns of the grid.

\subsection{Case 3: Calcarb}

Calcarb (http://www.calcarb.com) is a manufacturer of Carbon Bonded Carbon Fibre (CBCF) insulation material used in furnaces. Employing approximately 100 people, they produce a range of low and medium density carbon-based products from a Rayon fibre. Calcarb work closely with their customer base, and have developed technical partnerships with major clients in a number of sectors including aerospace, semiconductor, and automotive amongst others. The participants in the session were the company's Training Officer, Manufacturing Supervisor, Materials Manager and Process Improvement Manager.

As a manufacturing company and focussed very much on the engineering issues associated with the production of their insulation materials, Calcarb tend to take an informal approach to problem solving. Production issues are often solved on the shop floor or by individuals, although for more significant issues the management team would gather for whiteboard sessions. While conversant with tools such as Fishbone Diagrams, these sessions are not generally structured but instead used as a forum for individuals to share ideas. The use of the 
ICR Grid method was therefore an opportunity for the organisation to try a more prescriptive approach.

\subsubsection{Design problem}

The design problem addressed in the sessions was the marking of products for identification purposes through the manufacturing process. Calcarb have moulds that are shaped as board, cylinders or discs of various sizes that go through several drying and temperature processes before being machined to customer drawings. These machined parts can then be further processed. Previous attempts to identify the parts by etching, marking, painting and so on have proved ineffective, and so the design challenge in this instance was to try and develop alternative means to permanently identify them, with the main design constraints being durability, legibility, temperature resistance and contamination.

\subsubsection{Session output}

The output from the session is shown in Figure 6. After the problems caused by providing initial information items in the Scottoiler session, in the Calcarb session the participants were again given the freedom to choose in the first row of information resources. They were, however, asked to find something relating specifically to one of the design criteria as stated in the problem definition to ensure that there would be good diversity in the four threads. During the hour-long session, six rounds were completed. The session was again slow to start, with the manufacturing background of participants perhaps an initial barrier to the method. Information technology was also an issue, with the participants struggling to use OneNote and the tablet interfaces to various degrees.

Despite this, the session picked up after around 20 minutes, when a number of information items were sourced that provided new ways of approaching the identification problem, including one on a temperature resistant paint previously unknown to the team, and engendered greater enthusiasm for what might emerge from the session. It was at this point that participants also overcame a lack of fluency in sketching (again perhaps due to their background as manufacturing engineers) by focussing on text and annotation, meaning that the board began to take the form of a shared information resource. Participants found items, suggested how they would be used, and passed them on to others who would repeat this 
process. Although this was an unexpected use of the method, it seemed to suit the participants in this case.

\subsection{Feedback}

Qualitative data from the semi-structured interviews and observation were used to develop an understanding of how the method was used in the different cases. The format of the semistructured interview covered the topics of context, engagement, information use, communication, and output. An interview transcript from each interview session was created and reviewed, with the results summarised in Table 2.

\section{Analysis}

The comparative study described in Section 2.1 identified the ICR Grid as producing concepts of greater quality, variety and detail when compared to the 6-3-5 Method in controlled conditions. While issues relating to current practices of each company in concept design have been outlined in Section 3. the industrial applications provided only comparison across the sessions. A range of metrics were developed to achieve this and the results are set out in Table 3. This includes a breakdown of each grid in terms of: the number of rounds completed; the number of concepts and number of information items completed; the average sketch detail (using a scale of complexity adapted from Rogers et al. (2000) and information item detail (based on annotation and application of hyperlinks); the rate of positive response (number of positive responses divided by number of negative responses); the number of threads and average length of thread. It was found the ICR Grid performed well in the three different contexts, despite the issues regarding the usability of the OneNote interface. Participants acknowledged the potential benefits in conducting all their concept design work in an integrated environment, particularly the recording of pertinent information sources and the contextualisation of them by linking them to sketches. A diagrammatic representation of each grid is shown in Figure 7, with some key findings and insights described in the context of each.

LAT5 $6^{\circ}$ differed from the other two sessions in that there were only two participants. The simpler coordination meant that in this session the most rounds were completed in the allotted hour (8). The participants seemed to have fairly strong shared, pre-conceived notions of how the design should develop. This is evidenced by the speed with which the material loop 
principle was settled upon and embodied, despite there being two 'no' decisions in Thread A. This is similarity is illustrated in the sample from Figure 7(a). Based on the experience across the sessions, the preferred number would be three to six to allow adequate diversity without becoming overly cumbersome.

Scottoiler was the highest performing session on a number of counts. While the detail of concepts produced was comparable to the LAT56 $6^{\circ}$ session, the detail of the information sources retrieved was higher (1.92). This is a reflection of the fact that the team were comfortable with the subject area and using digital media to locate and apply them. The grid also had the longest threads (an average of 4.57 rounds), which tallies with the fact that they had the highest rate of positive response in evaluation. These longer threads afforded the opportunity to develop and expand on concepts, with Figure 7(b) illustrating how towards the end of a thread increasingly detailed information use and concept embodiment was being undertaken. The framing of the initial design problem was also revealed to be critical: it is recommended that a design problem is broken into key elements and these used as the initial inspiration for each thread of development. While maintaining flexibility, this ensures that a range of issues are addressed during a session.

Calcarb used the method in a way which differed significantly from the other two sessions. It was found that in this instance the participants were less comfortable sketching, and that the grid ultimately formed a matrix of information sources and suggested uses rather than sketches. This resulted in a significantly lower score for sketch detail (1) and is illustrated in Figure 7(c), which shows how the team were essentially using the grid to find and link relevant information, and make shared notes on how they could be applied. This in itself is a valuable resource for further development, and could conceivably be passed to a designer to use in the generation of solutions. It also suggests possibilities for developing the method as a way to facilitate collaborative Internet searching - something which until now has predominantly been undertaken by individuals. The IT problems encountered by participants in this session meant that the fewest number of rounds were completed (6) and also contributed to the lower score for information detail (1.38). This suggests that a more userfriendly interface would be desirable. The optimal solution would be a computer-based version operating on the company premises - this would afford flexibility to fit with working practices, increased comfort, and access to company resources. Development of such a bespoke system is an identified future area of work. 


\section{Conclusions}

This paper has outlined the application of the ICR Grid in a number of industrial settings. The three different companies (LAT 56 ${ }^{\circ}$, Scottoiler and Calcarb) invited to use the method provided varied feedback on its effectiveness. As highly aware design consultants, LAT $56^{\circ}$ quickly adapted to the rationale of the method and were able to apply it to a very specific design problem, although its diversity was inhibited by the fact there were only two participants. Scottoiler found the interactive benefits important, allowing them to improve communication across their R\&D team, and highlighted the importance of framing the initial problem correctly. The Calcarb session illustrated how the method could be recalibrated for team-based research exercises. The fact that the different companies were able to adapt the method to best suit their needs has allowed a number of insights to be drawn on future development and further application. It is anticipated that the principles of information use embodied in the method will be of interest to organisations engaged in concept work, as well as the Digital Library and Information Management communities.

\section{Acknowledgements}

The authors would like to thank LAT56 ${ }^{\circ}$, Scottoiler and Calcarb for their support, and to all participants who took part in the studies.

\section{References}

BENAMI, O. \& JIN, Y. Year. Creative stimulation in conceptual design. In: ASME 2002 Design Engineering Technical Confereces and Computer and Information in Engineering Conference, 2002 Montreal, Canada. DETC2002/DTM-34023.

CASAKIN, H. P. 2007. Factors of metaphors in design problem-solving: Implications for design creativity. International Journal of Design, 1, 21-33.

CHUANG, Y. \& CHEN, L. L. 2008. How to rate 100 visual stimuli efficiently. International Journal of Design, 2, 31-43.

CROSS, N. 1994. Engineering Design Methods, Strategies for Product Design, Chichester, UK, John Wiley \& Sons.

CROSS, N. 2004. Expertise in design: an overview. Design Studies, 25, 427-441.

DEMIAN, P. \& FRUCHTER, R. 2009. Effective visualisation of design versions: visual storytelling for design reuse. Research in Engineering Design, 19, 193-204.

DORST, K. \& CROSS, N. 2001. Creativity in the design process:co-evolution of problem-solution. Design Studies, 22, 425-437.

ERIS, O. 2004. Effective inquiry for innovative engineering design, Dordrecht, the Netherlands, Kluwer Academic Publishers.

FRENCH, M. 1985. Conceptual Design for Engineers, London, UK, The Design Council/ SpringerVerlag. 
GOLDSCHMIDT, G. 1991. The dialectics of sketching. Creativity Research Journal, 4, 123-143.

HELlFRITZ, H. 1978. Innovation via Galeriemethode (Innovation via the Art Gallery Method), Koenistein im Taunus, Germany, Eigenverlag.

KULKARNI, S., SUMMERS, J. D., VARGAS-HERNANDEZ, N. \& SHAH, J. J. 2001. Collaborative Sketching (C-Sketch) - An Idea Generation Technique for Engineering Design. The Journal of Creative Behavior, 35, 168-198.

OSBORN, A. 1953. Applied Imagination: Principles and Procedures of Creative Problem Solving, New York, NY, Charles Scribner's Sons.

PAHL, G. \& BEITZ, W. 1995. Engineering Design, A Systematic Approach, Bath, UK, Springer.

PUGH, S. 1991. Total Design, Reading, UK, Addison-Wesley.

RESTREPO, J. \& CHRISTIAANS, H. 2004. Problem Structuring and Information Access in Design. Journal of Design Research, 4.

RODGERS, P. A., GREEN, G. \& MCGOWN, A. 2000. Using concept sketches to track design progress. Design Studies, 21, 451-464.

ROHRBACH, B. 1969. Kreativ nach Regeln. Absatzwirtschaft, 12, 73-75.

SHAH, J. J., KULKARNI, S. V. \& VARGAS-HERNANDEZ, N. 2000. Evaluation of Idea Generation Methods for Conceptual Design: Effectiveness Metrics and Design of Experiments. Journal of Mechanical Design, 122, 377-385.

SHAH, J. J. \& VARGAS-HERNANDEZ, N. 2003. Metrics for measuring ideation effectiveness. Design Studies, 24, 111-134.

SIM, S. K. \& DUFFY, A. H. B. 2003. Towards an ontology of generic engineering design activities. Research in Engineering Design, 14, 200-223.

SMITH, S. M., KOHN, N. W. \& SHAH, J. Year. What you see is what you get: effects of provocative stimuli in creative invention. In: NSF International Workshop on Studying Design Creativity, $\begin{array}{lllll}\text { October } & 2008 & \text { Provence, }\end{array}$ http://mason.gmu.edu/ jgero/conferences/sdc08/papers/Smith.pdf (Accessed 12th June 2009).

SUTTON, R. I. \& HARGADON, A. 1996. Brainstorming groups in context: effectiveness in a product design firm. Administrative Science Quarterly, 41, 685-718.

ULRICH, K. T. \& EPPINGER, S. D. 1995. Product Design and Development, New York, NY, McGraw-Hill.

WODEHOUSE, A. J. 2010. Interactive Digital Support for Concept Design Teams. Doctor of Philosophy, University of Strathclyde. 


\section{Tables}

\begin{tabular}{llll}
\hline Company & Problem & Number & Background \\
\hline LAT56 & $\begin{array}{l}\text { Hanging suit carrier over a door } \\
\text { or rail }\end{array}$ & 2 & 2 Designers \\
Scottoiler & $\begin{array}{l}\text { Improved delivery of oil to } \\
\text { motorcycle chains }\end{array}$ & 4 & 3 Design Engineers \\
Calcarb & $\begin{array}{l}\text { Product for identification } \\
\text { throughout manufacturing process }\end{array}$ & 4 & 1 R\&D manager \\
& & 1 Training Officer \\
& & 1 Manufacturing Supervisor \\
& & 1 Materials Manager \\
& & 1 Process Improvement Manager \\
\hline
\end{tabular}

Table 1: Overview of the three industrial contexts 


\section{Topic}

The integrated environment appealed to the participants. They indicated that it was like 'logging into something and updating it', and provided a more robust record of their design work. The fact that user requirements were not addressed in depth was highlighted as a potential weakness, though it was acknowledged that detailed criteria and prior research could have formed grid information items.

Engagement

Participants commented that the concurrent ('all in the same pot') approach made the concept design process feel 'fresher', and helped to focus their design ideas. In terms of evaluating concepts during the session, they felt that 'usually a maybe' was a more appropriate than a definite yes or no, allowing aspects of concepts to be developed as they saw fit.

The information selected for use in Round 1 of the session was highlighted as critical. This dictated the direction of the threads and hence required careful consideration. Another observation was that the grid was 'organic' in that the type or depth of information required at a particular point was not dictated.

Communication Although fluent sketchers, the participants found verbal communication useful for clarification purposes. They preferred to do this rather than reinterpret unclear sketches, instead using any ambiguity as a discussion point to augment the development process

The participants were generally positive in their feedback, describing the grid as 'a good base to work from' and at least one idea was produced which had 'potential... to look into'. Although generally positive about the integrated nature of the development environment, they did observe that the method would benefit from a less complicated interface.
The team appreciated how the grid captured information as the session progressed, since the company currently struggles to document design meetings rigorously. Additionally, they enjoyed the variety provided by working on multiple ideas, finding it 'quite easy to deviate to another idea without getting caught up in the one thing'.

The team found the exchange of information between team members stimulating as it offered things you'd never seen before.' IT ability was also identified as important, with one participant inhibited by a lack of expertise. Searching activity was also rushed at times due to the timescales of the method and it was felt that this could compromise the quality of items.

Again the issues of Round 1 was raised, with participants suggesting that the proscribed initial sources detracted from the session as they would have chosen different paths for each.

The team felt that they tended to build on or change ideas rather than eliminate them, suggesting that if there is an aspect of a concept that does not seem feasible then they were liable to simply highlight or alter this aspect in order to 'let the next person have their input in as well.

Overall, the functionality of the integrated environment was well-received: 'I thought it was quite good you could drop a link in just like that... It was much more concise.' The results were felt to have been reasonably useful, with Thread 4 identified as having evolved particularly well. It was suggested with more careful identification of the starting point for the threads, the results could have been better.
Given a lack of regular team meetings, the participants returned some positive comments on the way the grid forced interaction with others' ideas. One highlighted the effectiveness of 'picking up somebody else's idea and researching it', with the evolution of concepts through evaluation, (information and reworking was identified as a useful approach.

It was apparent that for those unfamiliar with software and concept design techniques, the set-up and rules were still fairly complex, with one participant in particular struggling. Additionally, pace was again mentioned as an issue, and it was suggested that a longer-term approach over a period such as a half-day may be more effective.

A frustration at being able to find appropriate items was aired. The team did, however, find a number of items relating to paint manufacturers they were not previously aware of, and this was recognised as being particularly valuable knowledge for them moving forward.

Again, there was a reluctance to vote against ideas. Participants reported putting forward alternative ideas when voting yes rather than voting no: '...I found myself looking at the ideas and then trying to find a way that it could work... rather than not working.' The participant who struggled with the IT felt more comfortable in verbal communication.

There was a sense that the session showed a level of progression from start to finish: 'it takes you to that level where you can come out with maybe two ideas that are really good... and maybe points you in a direction.' The participants felt that the grid captured information sources they were not aware of and during the session they had managed to form 'an idea that we can take a step forward on.'

Table 2: Summary of feedback from post-session interviews 


\begin{tabular}{lllllllll}
\hline Company & Rounds & Concepts & $\begin{array}{l}\text { Info } \\
\text { sources }\end{array}$ & $\begin{array}{l}\text { Average } \\
\text { sketch } \\
\text { detail }\end{array}$ & $\begin{array}{l}\text { Average } \\
\text { info } \\
\text { detail }\end{array}$ & $\begin{array}{l}\text { Rate of } \\
\text { positive } \\
\text { response }\end{array}$ & $\begin{array}{l}\text { Number } \\
\text { of } \\
\text { threads }\end{array}$ & $\begin{array}{l}\text { Average } \\
\text { length of } \\
\text { thread }\end{array}$ \\
\hline LAT56 & 8 & 9 & 7 & 1.67 & 1.57 & 2.5 & 4 & 4 \\
Scottoiler & 7 & 15 & 17 & 1.69 & 1.92 & 4 & 7 & 4.57 \\
Calcarb & 6 & 10 & 12 & 1 & 1.38 & 2.5 & 3 & 4 \\
\hline
\end{tabular}

Table 3: Review of output 


\section{Figures}

A problem brief or

requirements document is

written with overall

context and problem aims

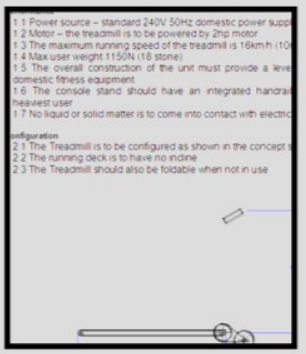

Number of participants

identified and time

allocated for session and

rounds

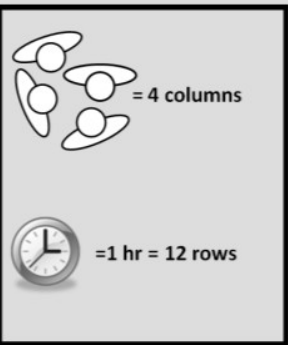

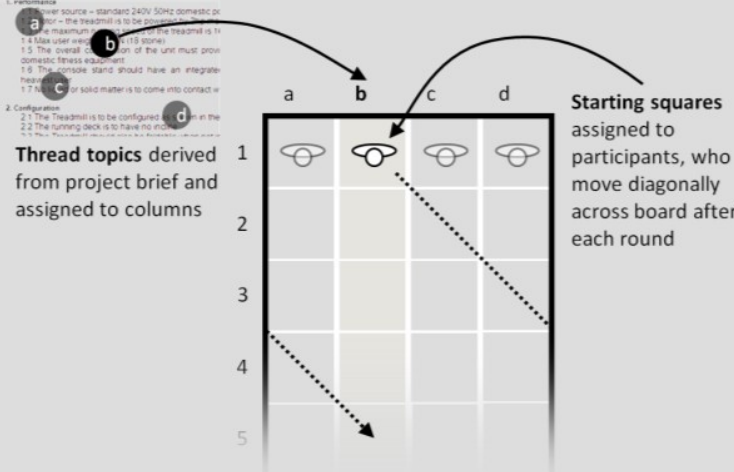

First round participants

search for information

related to thread topic

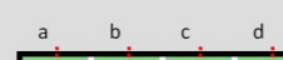

Second round participants sketch concept using

information item

1
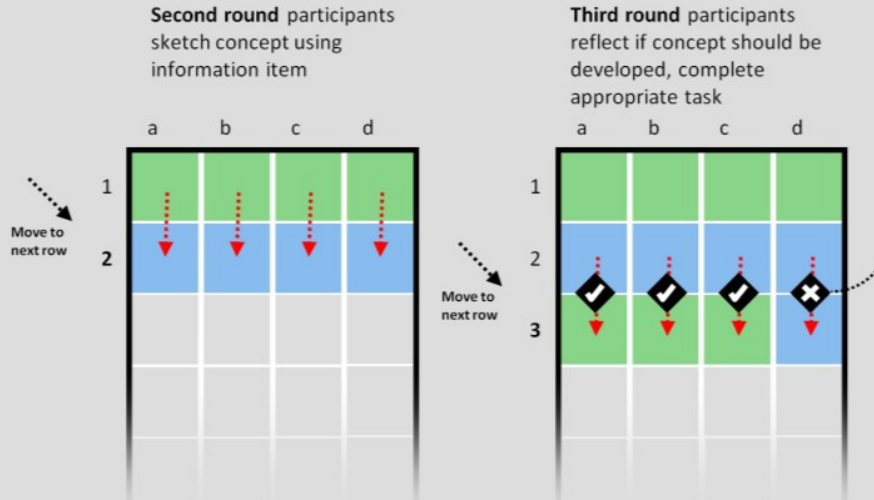

Third round participants

developed, complete

appropriate task

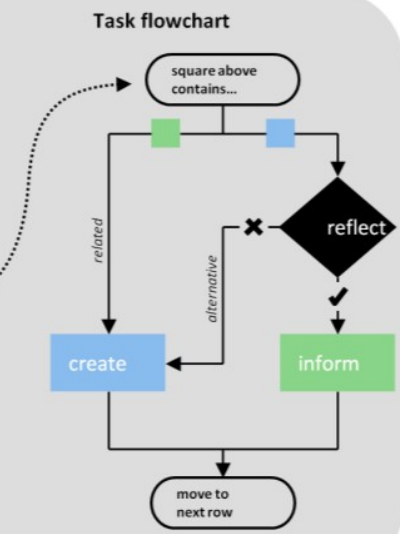

Grid evolves according to preceding squares and decisions

made, integrating ideas,

information and rationale

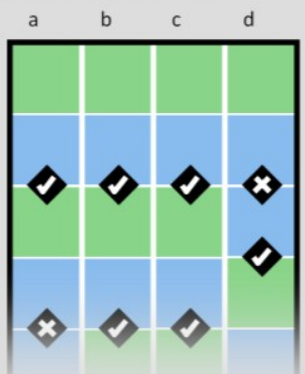

At end of session grid content reviewed for further use in design process

The grid is an interactive digital record of the team's interaction.

Information items are documented and linked for further use
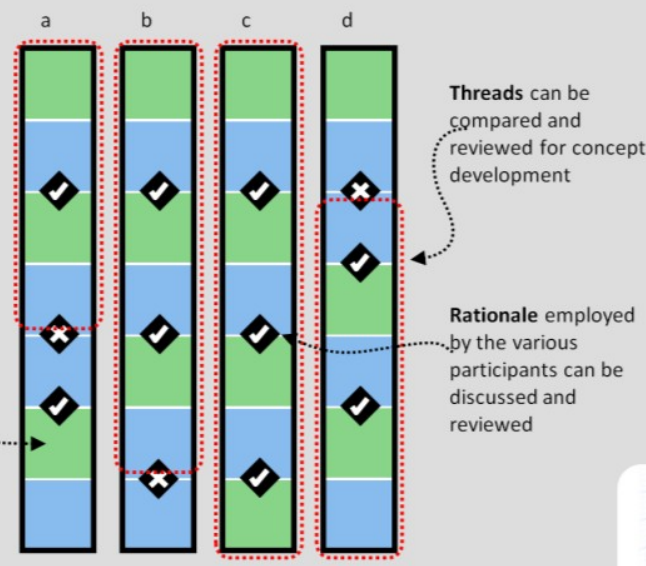

ICR Grid

Figure 1: Task flowchart and overview of grid composition 


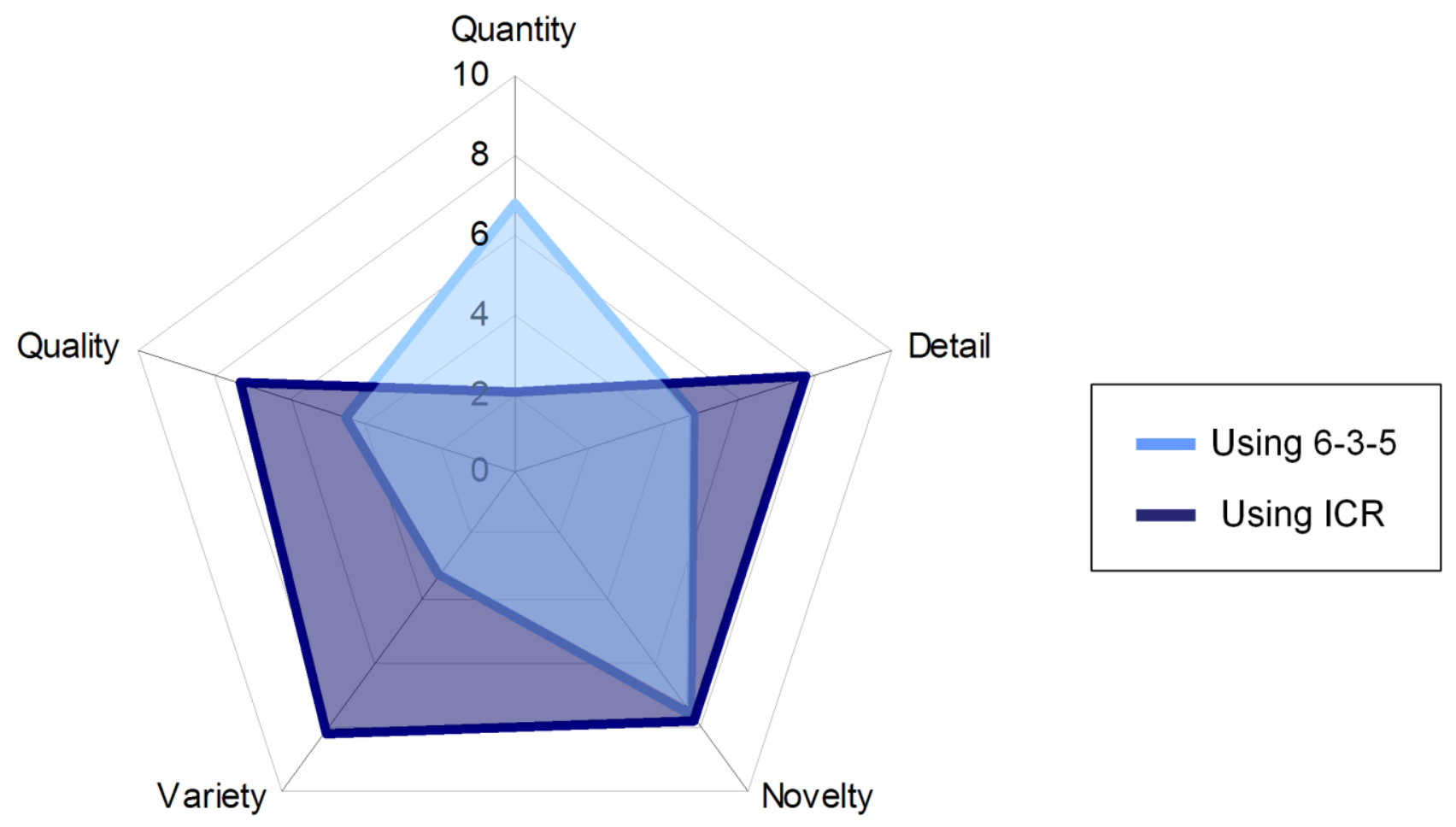

Figure 2: Comparison of the 6-3-5 Method and ICR Grid

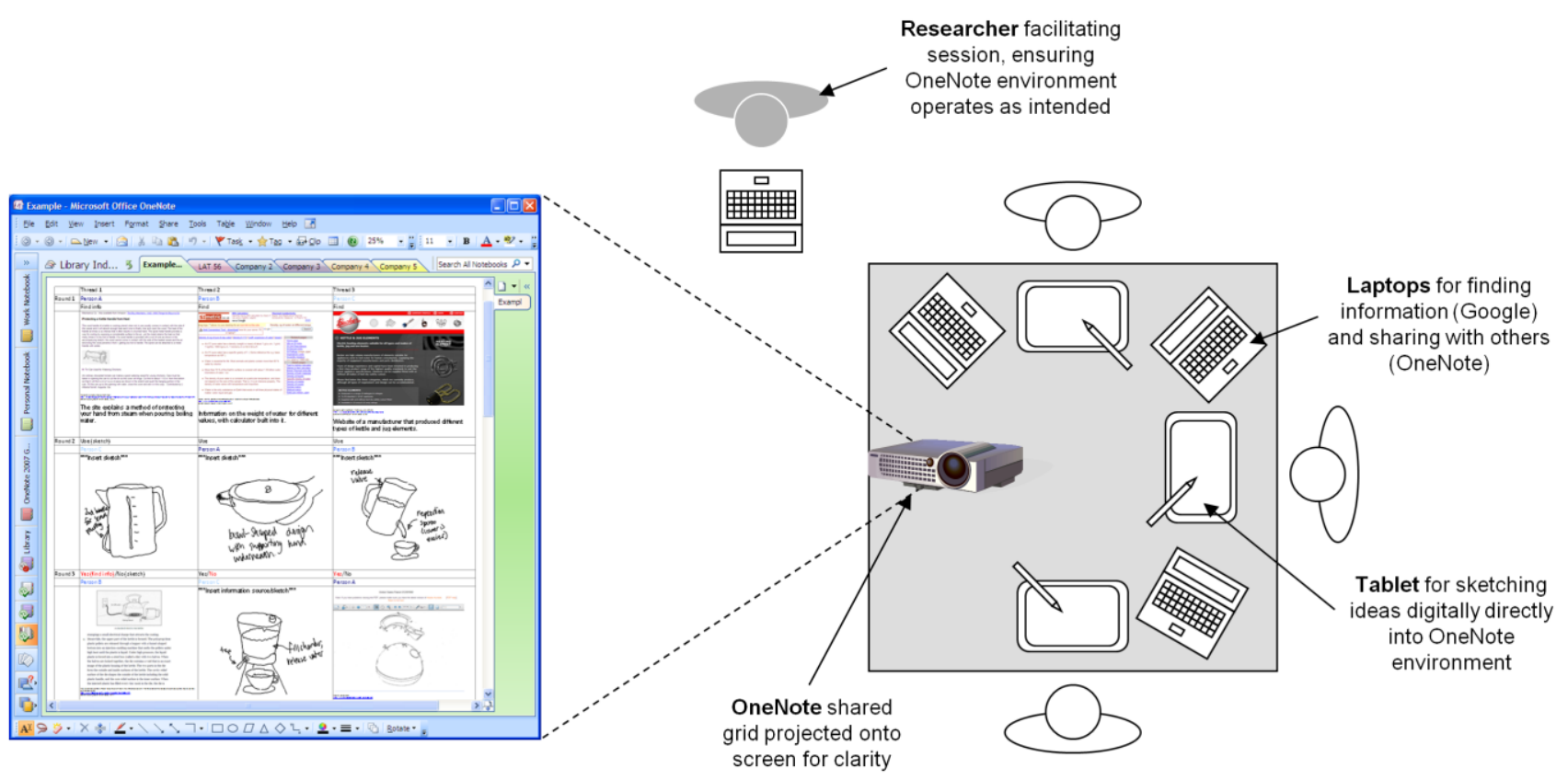

Figure 3: Set up for sessions 


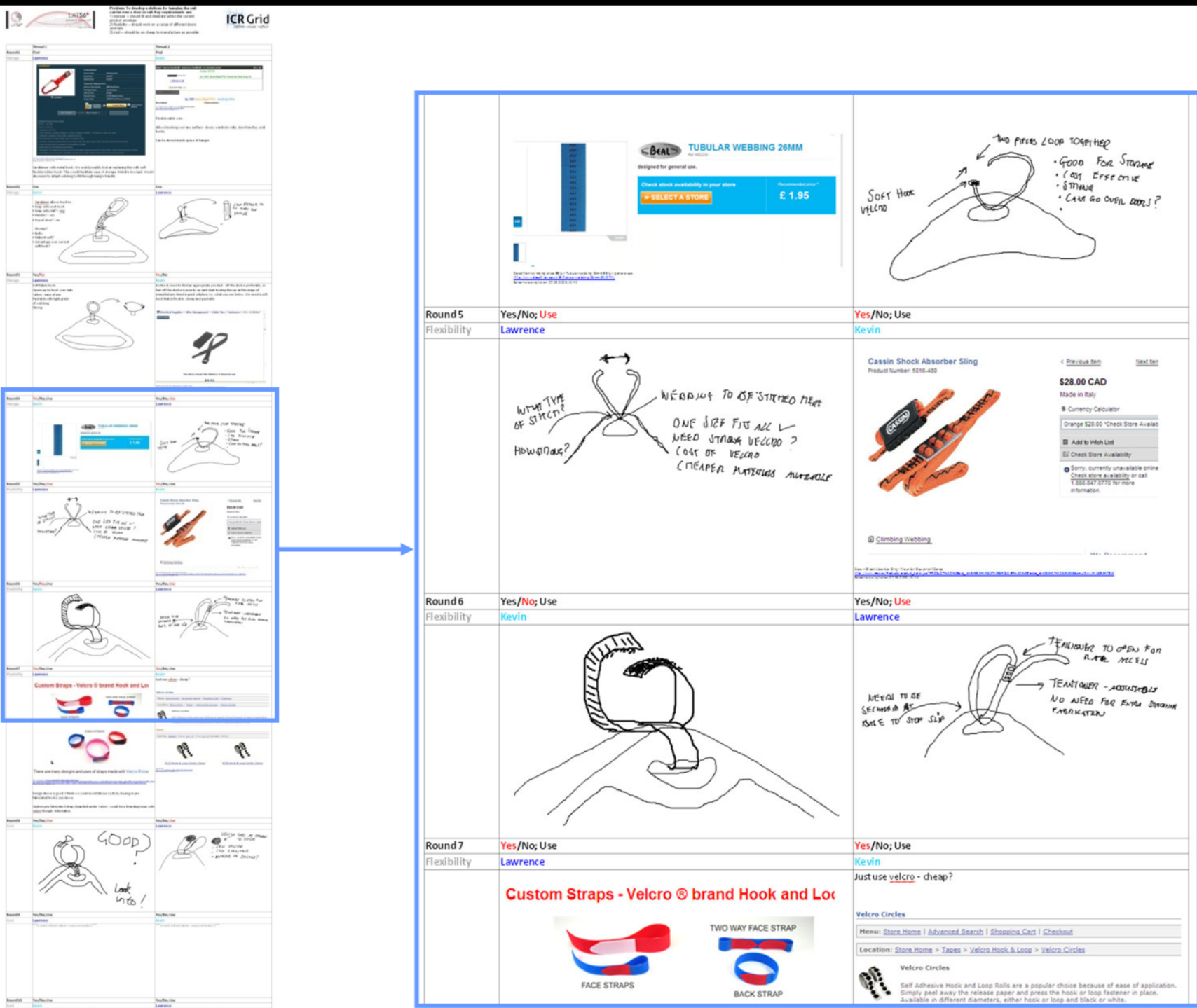

Figure 4: OneNote ICR Grid with sample from $\mathrm{LAT}^{\circ} 6^{\circ}$ design session 


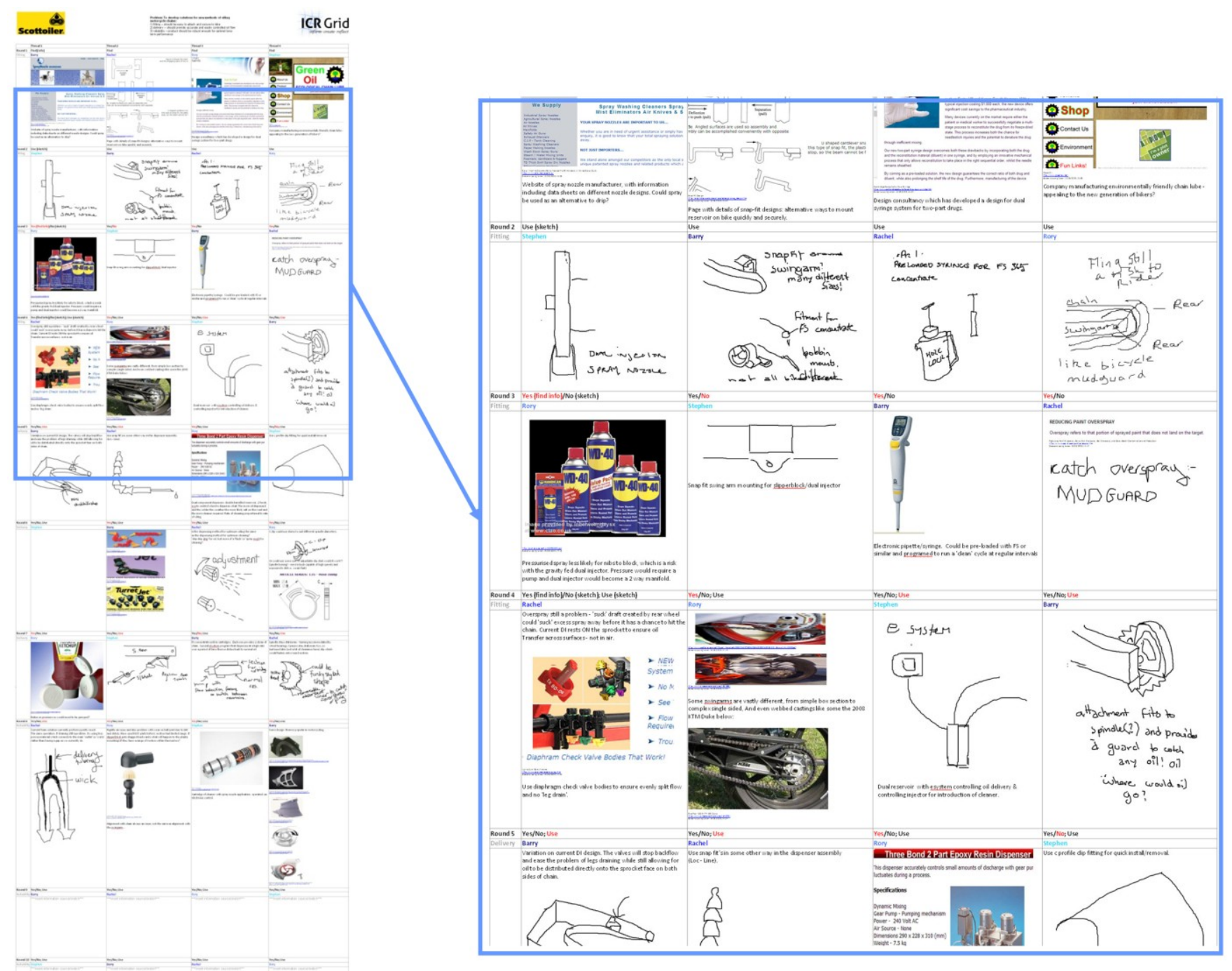

Figure 5: OneNote ICR Grid with sample from Scottoiler design session 


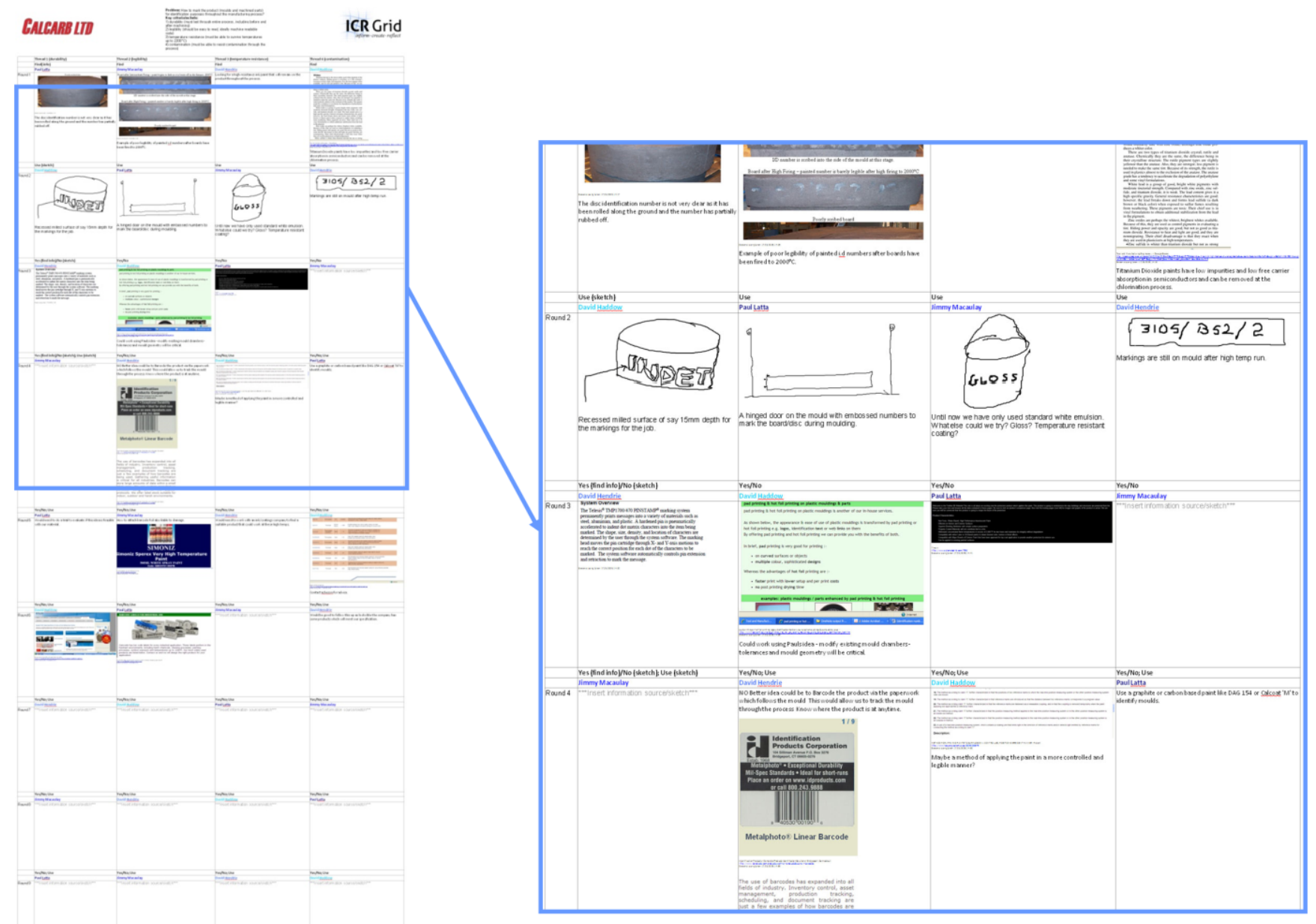

Figure 6: OneNote ICR Grid with sample from Calcarb design session 


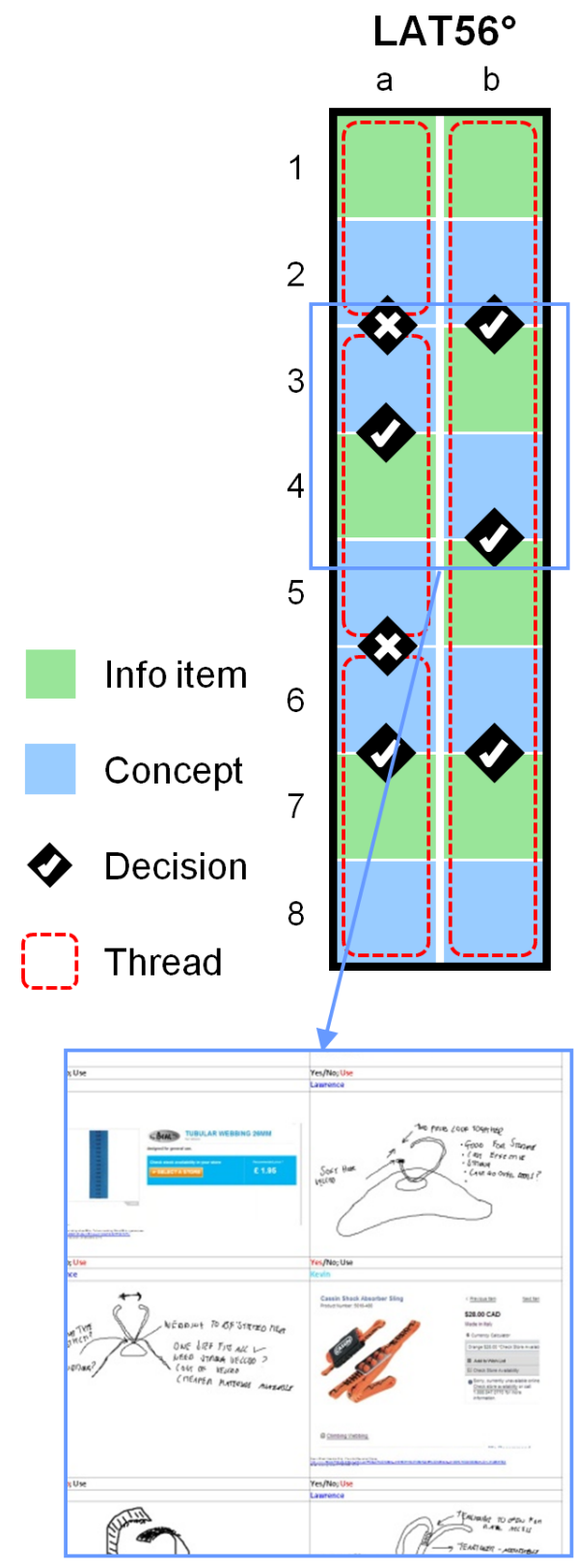

(a)

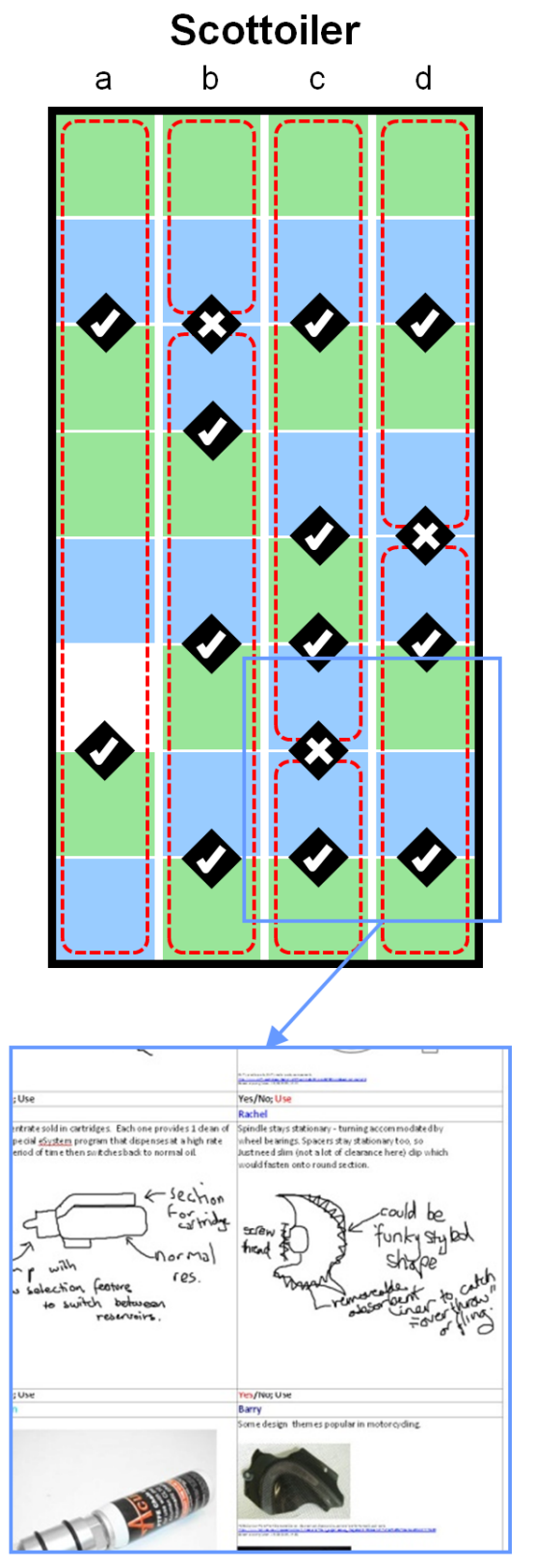

(b)

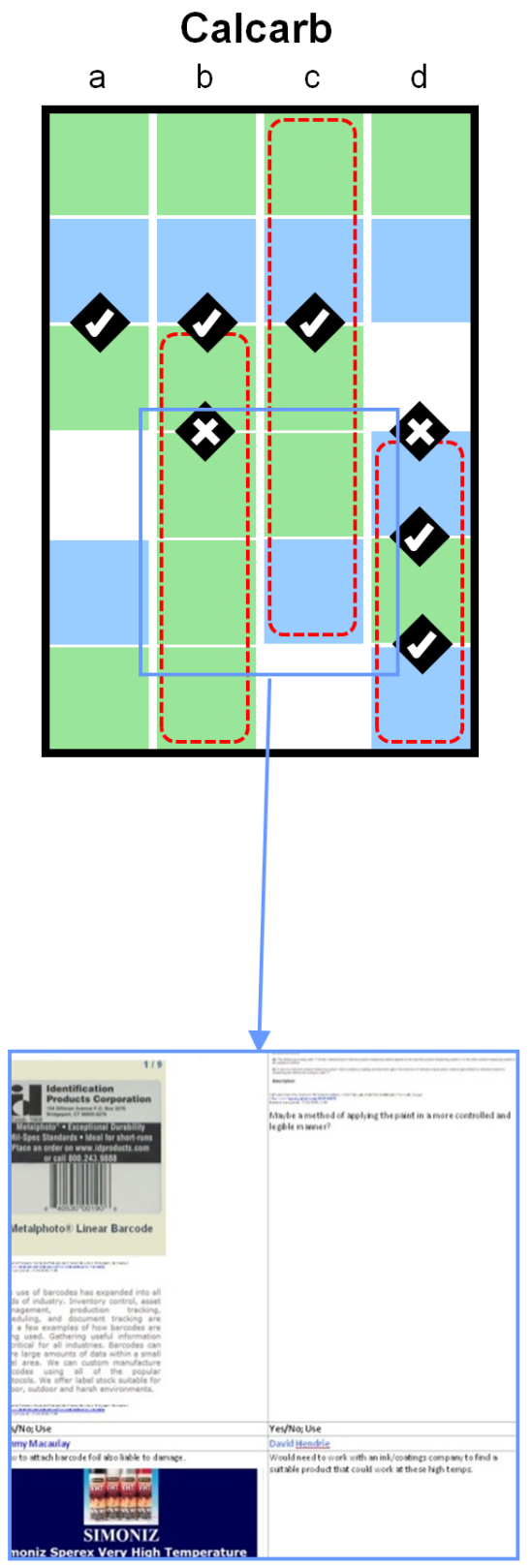

(c)

Figure 7: Analysis of output from the three sessions 